\title{
Electrical conductance of reconstructed silicon surfaces
}

\author{
Kwonjae Yoo $^{1}$ and H. H. Weitering ${ }^{2}$ \\ ${ }^{1}$ Department of Physics and Astronomy, The University of Tennessee, Knoxville, Tennessee 37996 \\ and Solid State Division, Oak Ridge National Laboratory, Oak Ridge, Tennessee 37831 \\ ${ }^{2}$ Department of Applied Physics and DIMES, Delft University of Technology, Lorentzweg 1, 2628 CJ Delft, The Netherlands
}

(Received 11 October 2001; published 8 March 2002)

\begin{abstract}
The electrical conductance of atomically clean, reconstructed silicon surfaces was studied as a function of temperature with macroscopic van der Pauw measurements in ultrahigh vacuum. The surface-state conductance of the $\mathrm{Si}(100) 2 \times 1$ surface was measured on a fully depleted silicon-on-insulator (SOI) substrate and on bulk $\mathrm{Si}$. The surface-state conductance has metallic temperature dependence, but its magnitude falls below the universal conductance quantum. The data furthermore reveal a clear signature of the $c(4 \times 2) \rightarrow 2 \times 1$ surface phase transition near $200 \mathrm{~K}$, which indicates that surface scattering increases with decreasing $c(4 \times 2)$ order on the surface. The surface conductance of $\operatorname{Si}(111) 7 \times 7$ was measured only on a SOI substrate. The temperature coefficient is metallic and the magnitude is larger than the universal conductance quantum.
\end{abstract}

DOI: 10.1103/PhysRevB.65.115424

PACS number(s): 73.25.+i, 68.35.Rh, 68.35.Bs

\section{INTRODUCTION}

Milestones in condensed-matter physics which have the greatest potential to impact the world, are often associated with the discovery of novel electronic-transport phenomena, frequently encountered in materials of considerable chemical and structural complexity or materials of reduced dimensionality. Examples abound, not the least of which date back to the birth of the transistor, or more recently high- $T_{c}$ superconductivity, giant magnetoresistance, and superconducting quantum-interference devices. Surfaces and ultrathin film materials represent a special class of low-dimensional systems that are not only interesting from the perspective of mesoscopic transport physics, but also due to their unique materials properties. Early studies of electronic transport in thin metal films have focused mainly on the transport physics. The seminal papers by Bergmann ${ }^{1}$ in the early 1980 s showed that the temperature- and magnetic-field dependence of the electrical conductance in polycrystalline films could be fitted to the theory of weak localization with compelling accuracy. Electronic transport studies of well-ordered surfaces and films of only a few monolayers thick are expected to be even more interesting as one tries to link the uniqueness of a material, i.e., atomic structure and (possibly lack of) band structure, to the measured transport properties down to the nanoscale. This approach obviously requires more sophisticated sample preparation and measurement under ultrahigh-vacuum (UHV) conditions.

The number of transport studies in UHV is rather limited, but a few groups in Japan and Germany have made significant contributions. Hasegawa and $\mathrm{Ino}^{2}$ monitored the resistance of a Si sample during deposition of a thin $\mathrm{Ag}$ film at room temperature (RT). The resistance decreases as expected, but the rate of decrease was strongly dependent on the reconstruction of the initial $\mathrm{Si}$ surface. They tentatively attributed the conductance change to the changes in the band bending beneath the surface, which in turn is determined by the spectrum of surface states near $E_{F}$. In other words, the subsurface conductance changes appreciably and the surfacestate conductance was assumed negligible. Attempts have also been made to measure the electrical conductance in surface states with a scanning tunneling microscope (STM). The surface-state conductance seems small. Estimates range from $10^{-6} \Omega^{-1} / \square$ to $10^{-8} \Omega^{-1} / \square$ for $\operatorname{Si}(111) 7 \times 7$ (Refs. 3 and 4) but the measurements were only done at RT. Others have measured electrical conductivity of thin epitaxial metal films on $\mathrm{Si}$ as a function of the thickness at fixed temperature, ${ }^{5,6}$ or as a function of the temperature at a fixed coverage. ${ }^{7,8}$ The parallel subsurface conductance was not considered in these studies, which seems justified at high coverage and at temperatures well below the "freeze-out" temperature of mobile bulk carriers.

Despite these significant contributions, the temperature dependence of the electrical conductivity has not yet been studied in the ultimate limit of a monatomic overlayer or reconstructed surface layer. In this paper we report on macroscopic, four-point probe measurements of the surface-state conductance of reconstructed $\mathrm{Si}(100)$ and $\mathrm{Si}(111)$ surfaces as a function of the temperature. Because it is not possible to electrically isolate the $\mathrm{Si}$ surface from its substrate, we explicitly take into account parallel conduction through the substrate and space-charge layer beneath the surface. Measurements on bulk samples were analyzed by calculating the space-charge layer contribution to the sheet conductance as a function of the temperature (see the Appendix). We also employed fully depleted $\mathrm{Si} / \mathrm{SiO}_{2} / \mathrm{Si}$ or silicon-on-insulator (SOI) material to minimize subsurface conduction. ${ }^{9,10}$ The SOI data are analyzed without relying on model-dependent space-charge layer calculations.

This paper explains in detail the measurements, calculations, and experimental procedures reported in an earlier paper $^{9}$ and also includes some other data on $\mathrm{Si}(111)$. The essential features of the reconstructed $\mathrm{Si}(100)$ and $\mathrm{Si}(111)$ surfaces will first be reviewed in Sec. II. Section III describes the experimental procedures. Results and discussions will be presented in Sec. IV. Section V summarizes the findings and concludes the paper. Calculations of the temperaturedependent carrier density and mobility in space-charge layers will be presented separately in the Appendix. This research has provided the first atomic scale surface characterization of 


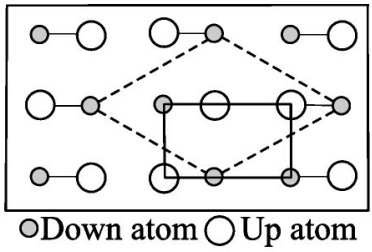

(a)

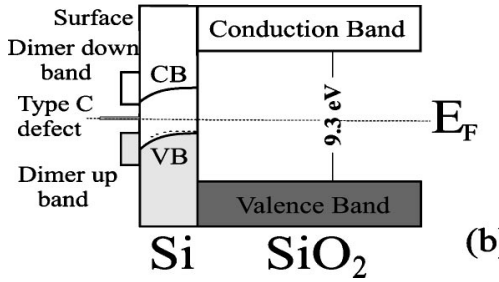

FIG. 1. (a) Top view of the $2 \times 1$ dimer array on $\mathrm{Si}(100)$. At RT, dimer atoms oscillate up-down, resulting in a time-averaged $2 \times 1$ structure (solid line). Dashed lines indicate the primitive unit cell of the $c(4 \times 2)$ structure. (b) Schematic band diagram of the SOI structure including the surface states and the type- $C$ defect state (not to scale).

(100) and (111) SOI material ${ }^{10,11}$ and temperature-dependent surface-conductivity measurement showing a clear signature of the $c(4 \times 2) \rightarrow 2 \times 1$ phase transition on $\operatorname{Si}(100){ }^{9}{ }^{9}$

\section{THE SURFACE OF Si(100) AND Si(111)}

The geometric and electronic structures of $\operatorname{Si}(100) 2 \times 1$ and $\mathrm{Si}(111) 7 \times 7$ are very well documented. ${ }^{12}$ Here, we summarize their properties that are relevant to the transport measurements.

The reconstructed $\mathrm{Si}(100)$ surface consists of a $2 \times 1$ array of buckled Si dimers atop the bulk-truncated surface [Fig. 1(a)]. ${ }^{12}$ The RT time-averaged $2 \times 1$ structure gradually transforms into an ordered $c(4 \times 2)$ superstructure below $200 \mathrm{~K}$ as flip-flopping Si dimers freeze into a static, "antiferromagnetic" array. This two-dimensional (2D) electron system is characterized by a $\sim 0.5 \mathrm{eV}$ gap separating the filled and empty surface-state bands, a total electron density of $6.78 \times 10^{14} \mathrm{~cm}^{-2}$. A parabolic fit of the surface-state band edge yields an effective mass of $\sim 0.4 m_{e}$. The Fermi level is pinned by defect-induced gap states associated with "type$C$ " surface defects. It is located at approximately $0.31 \mathrm{eV}$ above the valence band maximum (VBM) at the surface [Fig. 1(b)]. ${ }^{13}$

The $\operatorname{Si}(111) 7 \times 7$ surface is one of the very few "metallic" surface reconstructions. The reconstruction is a so-called "dimer adatom stacking (DAS) fault" or DAS reconstruction [Fig. 2(a)]. ${ }^{12}$ The "metallic surface state" is localized on the adatoms ${ }^{14}$ and has recently been mapped in $k$ space with high-resolution, angle-resolved photoemission. ${ }^{15}$ There are several narrow bands near $E_{F}$. Electron correlation effects appear important. Theoretical calculations indicate that the metallic behavior of $\mathrm{Si}(111) 7 \times 7$ is associated with only two electrons per $7 \times 7$ unit cell or $3.2 \times 10^{13} \mathrm{e} / \mathrm{cm}^{-2}$. The metallic surface state is responsible for the pinning of the Fermi level at approximately $0.65 \mathrm{eV}$ above the VBM [Fig.

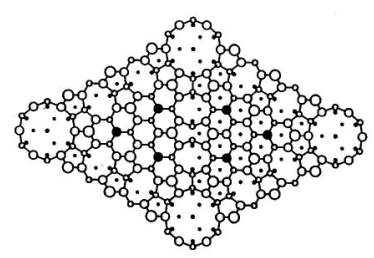

(a)

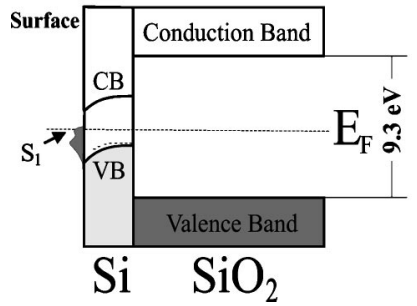

FIG. 2. (a) Top view of the $7 \times 7$ reconstruction on $\mathrm{Si}(111)$. (b) Schematic band bending diagram of $\operatorname{Si}(111) 7 \times 7$ including metallic surface states.

2(b) $].{ }^{16}$ There is no phase transition in the temperature range of the experiments.

\section{EXPERIMENTAL PROCEDURES}

We employed $n$-type $\operatorname{Si}(100)$ bulk wafer $(14 \Omega \mathrm{cm})$ and $p$-type SOI material. The SOI material was made via separation by implantation of oxygen (SIMOX). ${ }^{17}$ Commercial grade (100) SOI (19 $\Omega \mathrm{cm}$ ) was purchased from IBIS Technology Corporation. Research grade (111) SOI $(5 \Omega \mathrm{cm})$ was prepared at Spire Corporation. In short, SIMOX structures are prepared by a $1.8 \times 10^{18} \mathrm{~cm}^{-2} \mathrm{O}^{+}$-ion implantation at $200 \mathrm{keV}$, followed by annealing above $1500 \mathrm{~K}$ for 6 hours to regrow the $\mathrm{Si}$ and form buried $\mathrm{SiO}_{2}$. The resulting SIMOX materials typically consist of a 200-nm-thick crystalline Si layer on top of a 400-nm-thick layer of slightly substoichiometric $\mathrm{SiO}_{2} \cdot{ }^{18}$

The thickness of the Si top layer $t$ was reduced by dry oxidation and stripping with diluted HF. The purpose of the thinning procedure is to make sure that the physical thickness of the film is much less than the nominal depletion layer width $W=\sqrt{2 e \varepsilon_{0} \varepsilon_{r} v_{s} / N q}\left(\varepsilon_{0} \varepsilon_{r}\right.$ is the dielectric constant, $v_{s}$ the band bending, and $N$ the dopant level) ${ }^{10}$ In that case, the top layer will be fully depleted of mobile carriers and will be insulating below RT. The present doping levels are of the order of $10^{15} \mathrm{~cm}^{-3}$, hence $W \gg t .{ }^{10}$ The thickness of the remaining Si film was determined ex situ with Rutherford backscattering spectrometry and with ellipsometry. Both thickness measurements agree to within $10 \%$. In previous papers, we have shown using STM, low-energy electron diffraction (LEED), and Auger electron spectroscopy that the surface quality of the thinned SOI is comparable to that of bulk $\mathrm{Si} .{ }^{10,11}$ Thinner films can be prepared, but there is a risk that the thin film breaks up into islands. ${ }^{19}$

Ohmic contact pads $\left(2 \times 2 \mathrm{~mm}^{2}\right)$ for electrical measurements were defined by phosphorous- or boron-ion implant on the four corners of a $10 \times 10 \mathrm{~mm}^{2}$ sample for $n$-type and $p$-type $\mathrm{Si}$, respectively. The ion-beam energy was $30 \mathrm{kV}$ and 


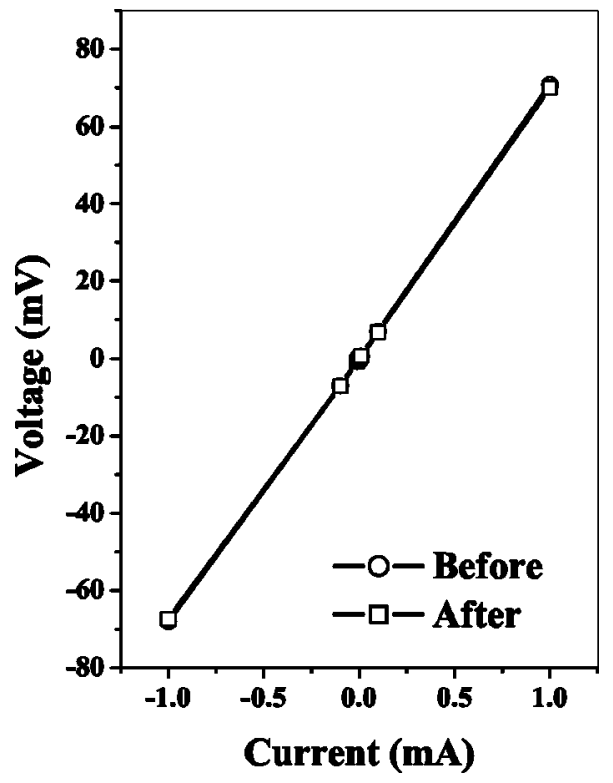

FIG. 3. $I-V$ curves of the Ohmic contacts before and after the temperature-dependent measurements in (100)SOI showing excellent reproducibility.

the ion dose was $\sim 10^{15} \mathrm{~cm}^{-2}$. The implantation depth was about $50 \mathrm{~nm}$ or less. The quality of the Ohmic contacts was confirmed by observing the linear voltage response to the applied electrical current before and after the temperaturedependent experiments. The linear $I-V$ curves were highly reproducible (Fig. 3). This reproducibility was never achieved without ion-implanted contact pads. We therefore believe that ion implantation is the preferred contact method for temperature-dependent conductivity measurements in UHV. Ta measurement probes were clamped onto the contact pads and the sample was transferred into the UHV chamber. Well-ordered $\mathrm{Si}(100) 2 \times 1$ surfaces were obtained by overnight degassing in UHV at $730 \mathrm{~K}$, followed by repeated flashing up to $1300 \mathrm{~K}$. We used direct-current heating. LEED patterns confirmed the formation of the $2 \times 1$ or $7 \times 7$ reconstruction.

Transport measurements were done as a function of the temperature using a dc current source. Data acquisition was fully computerized. The surface conductance $\Delta \sigma(T)$ of a bulk sample, in units of $\Omega^{-1} / \square$, is an excess quantity and is defined as follows: ${ }^{20}$

$$
G(T)=\sigma_{b}(T) d+\Delta \sigma(T),
$$

where $G(T)$ is the square conductance of the sample, $d$ is the thickness of the sample, and $\sigma_{b}$ is the conductivity of the bulk. $\Delta \sigma(T)$ includes electronic conduction through the surface states and through the space-charge layer beneath the surface and vanishes for an ideally truncated surface with no surface states. To obtain $\Delta \sigma$ for bulk $\operatorname{Si}(100) 2 \times 1$, we first measured the square conductance $G_{2 \times 1}(T)$ of the $n$-type bulk $\mathrm{Si}(100) 2 \times 1$ sample in UHV from $T \approx 120 \mathrm{~K}$ to $300 \mathrm{~K}$. Next, we saturated the clean $\mathrm{Si}(100) 2 \times 1$ surface with $\mathrm{O}_{2}$ at RT. This procedure destroys the reconstruction (as evidenced by a $1 \times 1$ LEED pattern), eliminates the surface states, ${ }^{21}$ and

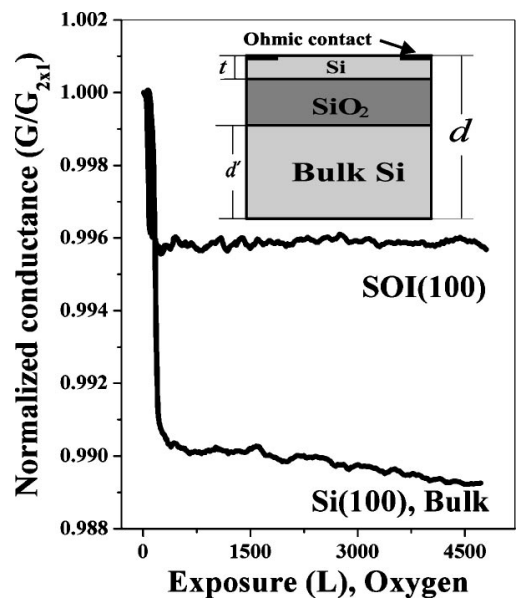

FIG. 4. Normalized conductance of an $n$-type bulk $\operatorname{Si}(100) 2$ $\times 1$ and a $p$-type $\mathrm{SOI}(100)$ during exposure to molecular oxygen at RT. Inset: Side view of the SOI sample with ion-implanted contacts.

creates a flat-band surface according to band bending measurements with Si $2 p$ core level spectroscopy. ${ }^{22}$ The conductance of the oxidized sample $G_{\mathrm{O}_{2}}$ was then measured from $120 \mathrm{~K}$ to $300 \mathrm{~K}$ and we obtained $\Delta \sigma(T)=G_{2 \times 1}(T)$ $-G_{\mathrm{O}_{2}}(T)$. In reality, $G_{\mathrm{O}_{2}}(T)$ is slightly less than $\sigma_{b}(T) d$ due to adsorbate-induced surface scattering, but we will show that this difference is negligible for $\mathrm{Si}$. The difference measurement to obtain $\Delta \sigma(T)$ is certainly prone to large errors if the contact resistances are not exactly reproducible after cooling and heating cycles because $G_{2 \times 1}(T)$ and $G_{\mathrm{O}_{2}}(T)$ differ by only a few percent. The use of ionimplanted contacts is therefore pivotal. Experiments were repeated several times to check the reproducibility of the $\Delta \sigma(T)$ values that are obtained via this subtraction procedure. Repeated measurements on the same sample yield values of $\Delta \sigma(T)$ that differ by less than $10 \%$. Comparisons between different samples show a larger spread (see, e.g., Fig. 4), which could perhaps be attributed to different surface qualities and/or different doping levels. Qualitatively, all data obtained from bulk $\mathrm{Si}(100)$ and various SOI samples exhibit the same trend upon exposure to $\mathrm{O}_{2}$ and upon ramping the temperature.

The oxidation was done by backfilling the chamber with molecular oxygen gas to a pressure of $1 \times 10^{-6}$ Torr. The oxygen pressure was measured with a vacuum gauge that was mounted such that the electrons and light from the gauge could not induce (photo)currents on the sample. All windows were covered during the transport measurements. Similar procedures were followed for the experiments on SOI.

\section{RESULTS AND DISCUSSION}

\section{A. Bulk Si(100)}

Figure 4 shows the normalized conductance of the $(2$ $\times 1)$-reconstructed bulk Si [and $(2 \times 1)$-reconstructed SOI sample] as a function of exposure to $\mathrm{O}_{2}$ at RT. It shows that a clean $(2 \times 1)$ reconstructed sample is less resistive than a sample passivated with $\mathrm{O}_{2}$, in agreement with other RT 


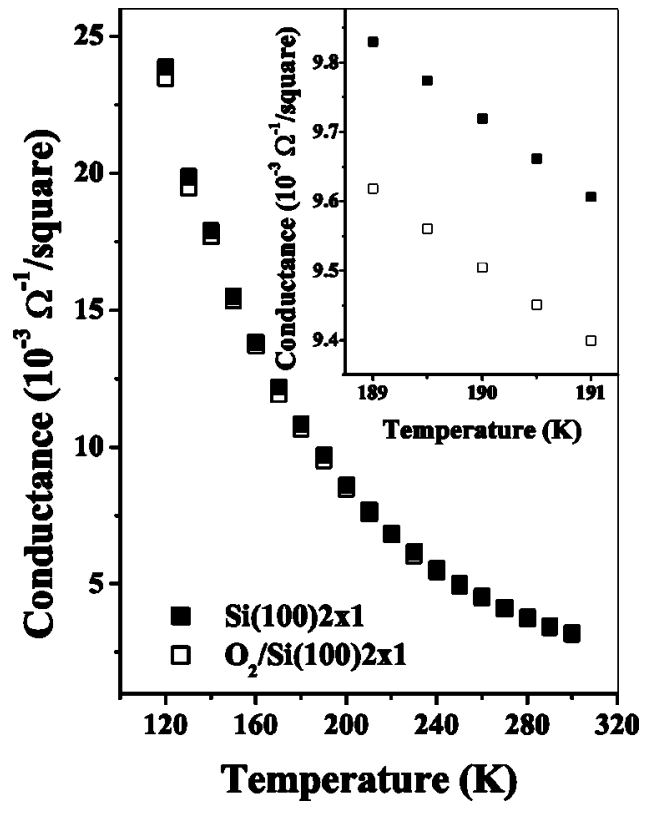

FIG. 5. Sheet conductance as a function of temperature for bulk $\operatorname{Si}(100) 2 \times 1$ and oxidized bulk $\mathrm{Si}(100)$. Inset: Enlarged scale showing the difference between the clean and oxidized $\mathrm{Si}(100)$. This difference amounts to $1 \%$ of total sheet conductance.

chemisorption studies. ${ }^{23,24}$ Wormeester et al. argued that the sheet conductance of $\mathrm{Si}(100) 2 \times 1$ is reduced during oxygen exposure because the "accumulation layer" beneath the surface gradually turns into a flat band system. ${ }^{23}$ Petersen et al. recognized that the space-charge layer beneath the $\operatorname{Si}(100) 2 \times 1$ surface is a depletion layer and not an accumulation layer. ${ }^{24}$ The space-charge layer should thus become less depleted (hence more conducting) during exposure to oxygen and formation of the flat band surface, which is in obvious conflict with the experimental data. Petersen et al. offered an alternative explanation. They attributed the reduction of the sheet conductance to diffusive surface scattering due to the oxygen-induced roughening of the surface. The surface-state contribution was deemed negligible in both studies.

A simple argument shows that this diffuse scattering effect is small and does not reverse the sign of $\Delta \sigma(T)$. In the abrupt depletion approximation, ${ }^{33}$ extrinsic carriers originating from the $(2 \times 1)$ depletion layer (width $\sim L_{D}$ ) are trapped in the surface states. If we assume that the surface states do not conduct, we obtain $G_{2 \times 1} \approx \sigma_{b}\left(d-L_{D}\right) ; L_{D}$ is the Debije screening length (see the Appendix). In the limit of completely diffuse scattering at the flatband $\mathrm{O}_{2} / \mathrm{Si}(100)$ surface, one obtains $G_{\mathrm{O}_{2}} \approx \sigma_{b}\left(d-\lambda_{b}\right)$, i.e., there is a "stagnant" layer of thickness $\lambda_{b}$ beneath the surface where $\lambda_{b}$ is the bulk mean free path. Since $\lambda_{b} \ll L_{D}$ for Si at RT and below (see the Appendix), $\Delta \sigma(T)=\left[G_{2 \times 1}(T)-G_{\mathrm{O}_{2}}(T)\right]$ $<0$, in contradiction with experiment. The only plausible explanation for the large conductivity of the $(2 \times 1)$-reconstructed surface seems to be the presence of a surface-state conduction channel, which is gradually destroyed by the adsorption of $\mathrm{O}_{2}$.

Figure 5 shows the temperature-dependent sheet conduc-

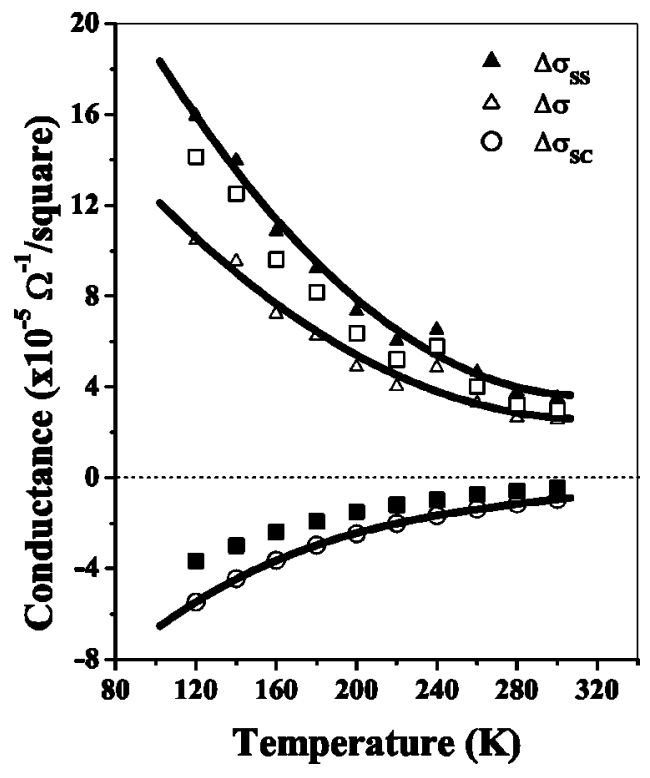

FIG. 6. Experimental surface conductance $\Delta \sigma(T)$ of the $n$-type bulk $\mathrm{Si}(100)$ sample. Squares represent the space-charge-layer conductance (filled) and surface-state conductance (open), assuming a residual band bending at the oxidized surface of $0.1 \mathrm{eV}$.

tance of bulk $\mathrm{Si}(100) 2 \times 1$ and of oxidized $\mathrm{Si}$. Both curves are almost identical and show a decreasing conductivity with increasing temperature, which is due to phonon scattering in the bulk. On the enlarged scale (inset), however, the conductance of the $(2 \times 1)$-reconstructed sample is always larger than that of the oxidized surface. This behavior, consistent with the RT data of Fig. 4, was very reproducible. We now obtain the experimental surface conductance $\Delta \sigma(T)$ $=G_{2 \times 1}(T)-G_{\mathrm{O}_{2}}(T)$, which is shown in Fig. 6 .

In order to separate the surface-state conductance $\Delta \sigma_{s s}(T)$ from the total surface conductance $\Delta \sigma(T)$, we subtract the space-charge layer contribution $\Delta \sigma_{s c}(T)$ that is calculated in the Appendix. Briefly, $\Delta \sigma_{s c}(T)$ is derived from the Boltzmann transport equation in a transverse electric field associated with the space-charge layer. ${ }^{20}$ The carrier mobility in the space-charge layer is furthermore reduced due to diffusive surface scattering. This effect is treated phenomenologically by means of the Fuchs-Sondheimer parameter $r(T)$ that represents the fraction of specular scattering events $[0 \leqslant r(T) \leqslant 1] .{ }^{20,25}$ Assuming that there are no other surface channels besides the space-charge layer and the surface states, we obtain $\Delta \sigma_{s s}(T)=\Delta \sigma(T)-\Delta \sigma_{s c}(T)$. The crucial observation is that $\Delta \sigma_{s c}(T)$ and $\Delta \sigma(T)$ always maintain opposite sign over the entire temperature range of the experiment, regardless of the precise value of the phenomenological Fuchs parameter and regardless of whether the bands at the oxidized surface are perfectly flat or slightly bent. As mentioned in the Appendix, the value of $r(T)$ hardly matters as the corrections to $\mu_{s}$ and $\Delta \sigma_{s c}$ remain within $1 \%$ or so. In Fig. 6, we included the calculations for bulk Si assuming a residual band bending of $0.1 \mathrm{eV}$ at the oxidized surface. It is clear that this reduces the magnitude of the surface-state conductance, but this still does not affect our conclusion, i.e., $\Delta \sigma_{s c}(T)<0, \Delta \sigma(T)>0$, and $\Delta \sigma_{s s}(T)>0$. Our data are 


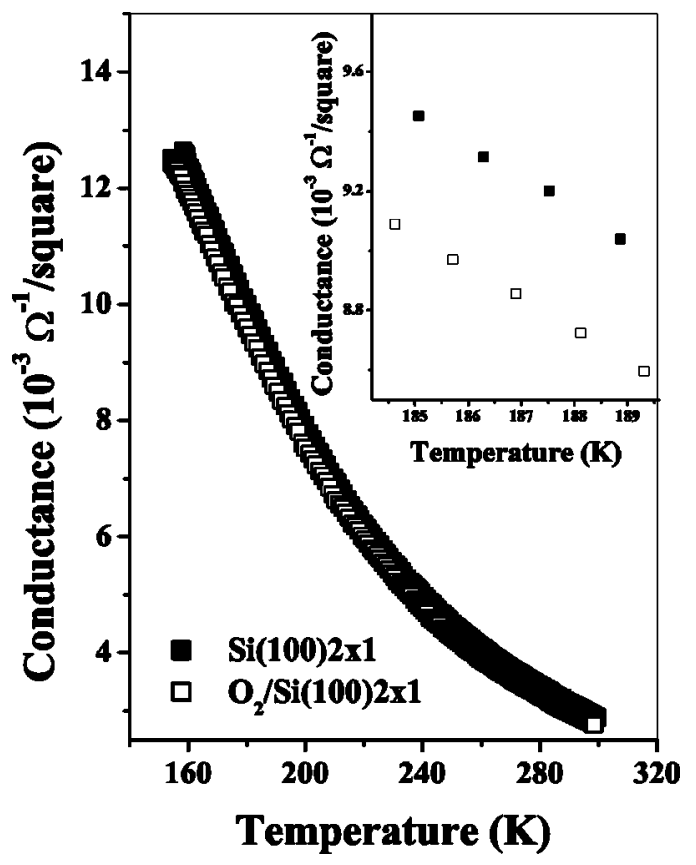

FIG. 7. Total sheet conductance of $\mathrm{Si}(100) 2 \times 1$ and oxidized $\mathrm{Si}(100)$ on SOI as a function of temperature. The Si layer is $90 \mathrm{~nm}$ thick. Inset: Enlarged scale showing the difference between the clean and oxidized $\mathrm{Si}(100)$.

fully consistent with the presence of a surface-state conduction channel on $\mathrm{Si}(100) 2 \times 1$ that is gradually destroyed by oxidation. The data furthermore show that $\Delta \sigma_{s s}(T)$ decreases with increasing temperature, which is indicative of metallic behavior. A discussion about the meaning of this "metallicity" will be deferred to the following section.

\section{B. (100)SOI}

Another experiment was carried out to determine the surface-state conductance without resorting to temperaturedependent (and model-dependent) space-charge layer calculations and assumptions about the mobility reduction beneath the $\mathrm{O}_{2}$-passivated surface. In this experiment, a 90-nm-thick, fully depleted $p$-type SOI sample was used. The conductance of the $(2 \times 1)$-reconstructed (100)SOI also decreases upon exposure to molecular oxygen, similar to the bulk sample (Fig. 4). Temperature-dependent measurements of the clean and oxidized SOI samples are shown in Fig. 7. Even though there is a buried-oxide layer, the resistance between the top layer and bottom substrate was nevertheless finite $(\sim 1 \mathrm{M} \Omega)$. It is a drawback in our experiment that the substoichiometric subsurface oxide of SOI is not perfectly insulating. ${ }^{26}$ Substantial leakage current still flows through the bottom substrate. However, the oxidation of the top layer is not expected to affect the conductance through the substrate beneath the 400-nm-thick buried oxide. Therefore, we model the SOI as a parallel-plate conductor separated by a buried oxide (Fig. 4, inset), and obtain $G_{2 \times 1}(T)=\sigma_{b}(T) d^{\prime}$ $+\Delta \sigma_{s s}(T)$ and $G_{\mathrm{O}_{2}}(T)=\sigma_{b}(T)\left[d^{\prime}+t-(1-r) \lambda_{b}(T)\right]$, giving

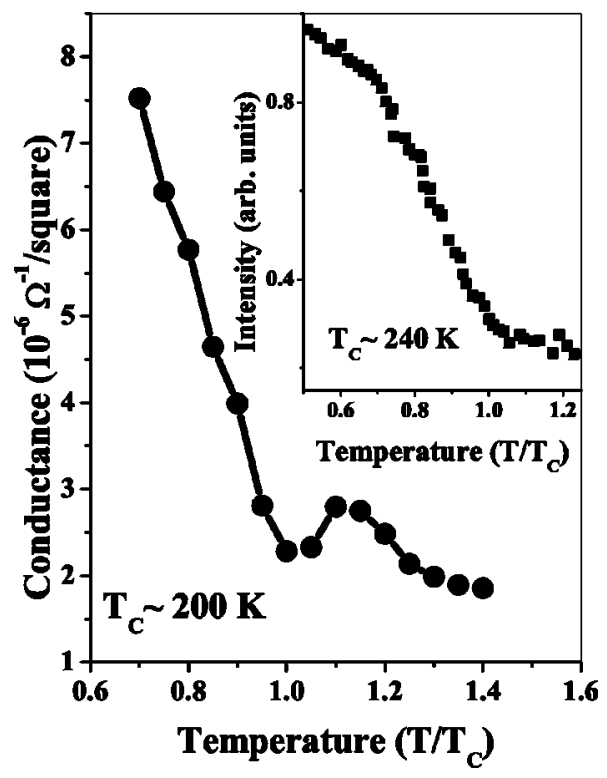

FIG. 8. Experimental determination of the surface-state conductance of (100) SOI, using Eq. (2) with $r=0$ (diffusive scattering). The intensity of the $(3 / 2,3 / 4)$ LEED spot of the $c(4 \times 2)$ superstructure is taken from Ref. 27.

$$
\Delta \sigma_{s s}(T)=G_{2 \times 1}(T)-G_{\mathrm{O}_{2}}(T) \frac{d^{\prime}}{d^{\prime}+t-(1-r) \lambda_{b}(T)},
$$

$G_{2 \times 1}(T)$ and $G_{\mathrm{O}_{2}}(T)$ are the square conductances from the clean and passivated SOI wafers, respectively; $t$ and $d^{\prime}$ are the thickness of the Si top layer and the supporting substrate, respectively, and $r$ is the Fuchs' specularity parameter of the $\mathrm{O}_{2}$-passivated surface. Note that $\lambda(T)<t \ll d^{\prime}$ for $T$ $>120 \mathrm{~K}$. The surface-state conductance $\Delta \sigma_{s s}(T)$ is obtained from Eq. (2) and is shown in Fig. 8 using $r=0$ (diffuse scattering). The overall behavior appears rather well reproducible during different experimental runs. In reality, $r$ is a model-dependent parameter that varies with temperature, which also makes $\Delta \sigma_{s s}(T)$ somewhat model dependent. However, its most salient feature, i.e., the sharp increase below $200 \mathrm{~K}$ is model independent because $d^{\prime} \gg t, \lambda(T)$. Possible residual band bending at the oxidized surface should not affect the overall behavior shown in Fig. 8. Even if the oxidized film were fully depleted due to residual band bending, we would obtain $\Delta \sigma_{s s}(T)=G_{2 \times 1}(T)-G_{\mathrm{O}_{2}}(T)$. This is very slightly different from the curve in Fig. 8, but this difference is too small to be resolved on the scale of this figure.

The experiment clearly shows that the surface-state conduction increases rapidly below 200-220 K. This behavior is also evident from the bulk measurements in Fig. 6 although the inflection of the conductivity curves near $200 \mathrm{~K}$ is much less clear for the bulk sample. We speculate that $\Delta \sigma_{s s}(T)$ decreases with increasing temperature as the buckled dimers on $\mathrm{Si}(100)$ gradually lose their long-range $c(4$ $\times 2$ ) ordering. This order-disorder transition is almost complete near $200 \mathrm{~K},{ }^{27}$ which would explain why $\Delta \sigma_{s s}(T)$ 
reaches a minimum (maximum surface scattering). To illustrate this point, we also show the intensity of the $(3 / 2,3 / 4)$ spot in LEED as a function of $T / T c$ as reported in Ref. 27, which reflects the order parameter of the $c(4 \times 2)$ reconstruction. This figure strongly suggests a link between the surface-state conductance and structural (dis)order on the surface. Our own LEED patterns also showed the $c(4 \times 2)$ superstructure at the lowest measurement temperature (120 $\mathrm{K})$. At RT, there were only $2 \times 1$ spots.

The temperature dependence of the surface-state conductivity is indicative of metallic behavior whereas the $(2 \times 1)$ and $c(4 \times 2)$ reconstructions have a $\sim 0.5 \mathrm{eV}$ surface-state band gap. ${ }^{12}$ Because of this gap, it is unlikely that $\mathrm{Si}(100) 2 \times 1$ conducts like a metal. Consider the absolute magnitude of the surface-state conductance. It is of the order of $\sim 10^{-6} \Omega^{-1} / \square$. Assuming that the Boltzmann theory is applicable, then one can write for a two-dimensional conductor $^{28}$

$$
\sigma=\frac{e^{2}}{h} k_{F} l .
$$

The Ioffe-Regel limit for metallic conduction states that the mean free path $l$ cannot be shorter than $k_{F}^{-1}$ or, alternatively, the interatomic distance. ${ }^{28}$ The minimum metallic conductance in $2 \mathrm{D}$ is thus $\sigma_{\min }=3.83 \times 10^{-5} \Omega^{-1} / \square$, independent of carrier density. The surface-state conductance derived from the SOI measurements clearly falls below this value so that one should classify this surface as a "bad metal," 29,30 i.e., the temperature coefficient indicates metallic behavior but the mean free path has no physical interpretation. Interestingly, the absolute surface-state conductance derived from the bulk measurements exceeds the minimum conductance at low temperatures and levels off near RT at a value that is close to the minimum conductance. One should not emphasize these absolute values too much as they have been obtained in a rather indirect way (by oxidation and subtraction of two resistivity curves), but the order of the magnitude surely suggests that the mean free path is either very short or that the Boltzmann picture breaks down altogether. The different magnitude of the surface conductance on bulk $\mathrm{Si}(100)$ and SOI is possibly related to differences in the surface quality.

We finally note that the surface-state conductance is large enough to completely offset the conductivity reduction in the space-charge layer beneath the $2 \times 1$ surface so that the total surface conduction (i.e., surface states and space-charge layer) is always positive. Electronic transport in this 2D electron system likely involves excitations between the surfacestate bands and possibly the type- $C$ gap state, ${ }^{13}$ but evidently the dominant factor determining the temperature coefficient between $120 \mathrm{~K}$ and $300 \mathrm{~K}$ is the order parameter of the $c(4 \times 2)$ structure. We, therefore, attribute the decrease of the surface-state conductance up to $200 \mathrm{~K}$ to the gradual demise of long-range $c(4 \times 2)$ order on the surface.

\section{C. (111)SOI}

Figure 9 shows the normalized conductance of the (7 $\times 7)$-reconstructed SOI sample as a function of exposure to

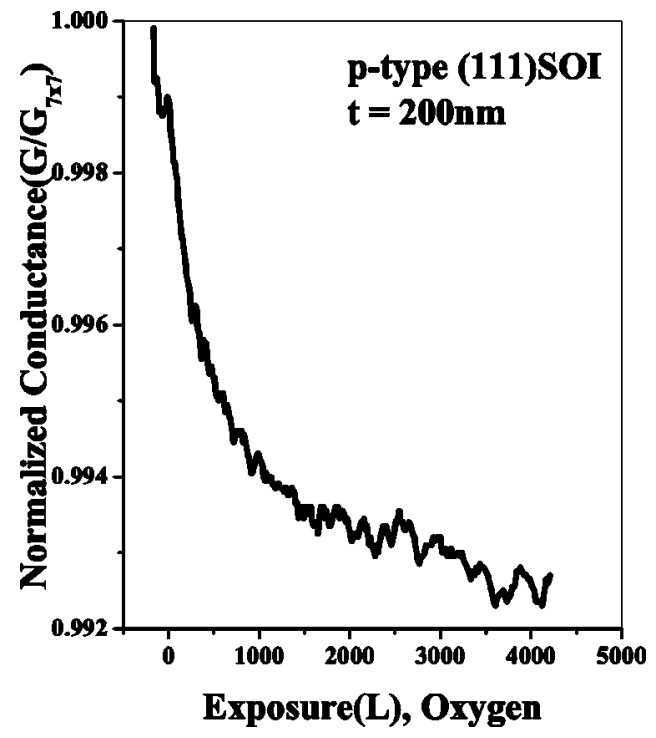

FIG. 9. Normalized conductance of $\operatorname{Si}(111) 7 \times 7$ as a function of oxygen exposure at RT. The data are recorded from an SOI sample with a Si top layer of $200 \mathrm{~nm}$.

$\mathrm{O}_{2}$ at RT. It shows that the clean, reconstructed $7 \times 7$ surface is a better conductor than the oxidized surface, which agrees with the STM point-contact measurements by Hasegawa, Lyo, and Avouris. ${ }^{3}$ Their interpretation is that the surface states of $\mathrm{Si}(111) 7 \times 7$ conduct and that this conduction channel is eventually destroyed by the oxidation of the surface. ${ }^{3}$ Our measurements are different from the four-point probe measurements by Petersen et al. on p-type bulk $\mathrm{Si}(111){ }^{24}$ Their measurements show a very steep rise in conductivity up to about $80 \mathrm{~L}$ of $\mathrm{O}_{2}$. It then decreases below the initial conductance as the exposure continues. Petersen et al. proposed that the initial increase in conductivity is related to the formation of a "molecular precursor" to oxygen dissociation. ${ }^{24}$ The existence of this precursor has been correlated with an unusual-low work function. In their interpretation, the low work function suggests that the Fermi level is located very close to the valence band maximum so that the depletion layer beneath the $7 \times 7$ surface may turn into a highly conducting accumulation layer. Our measurements, however, are inconsistent with theirs.

Figure 10 shows the total sheet conductance of $\mathrm{Si}(111) 7$ $\times 7$ and oxidized $\mathrm{Si}(111)$ on SOI as a function of temperature. The data seemingly overlap. However, on an enlarged vertical scale, one can clearly see that the curves are slightly different. The sheet conductance of the $7 \times 7$ reconstructed SOI sample is larger than that of the oxidized sample. This behavior is similar to that of $\operatorname{Si}(100) 2 \times 1$, which we discussed in the preceding section. The experimental surface conductance $\Delta \sigma(T)$ can be obtained by subtracting $G_{\mathrm{O}_{2}}(T)$ from $G_{7 \times 7}(T)$. The resulting curve in Fig. 11 looks similar to that of $\Delta \sigma(T)_{s s}$ for bulk $\mathrm{Si}(100)$. There are no clear indications of a phase transition. It shows that $\operatorname{Si}(111) 7 \times 7$ is a metallic surface. The conductance is larger than that of $\mathrm{Si}(100)$ and well above the fundamental conductance quantum. 


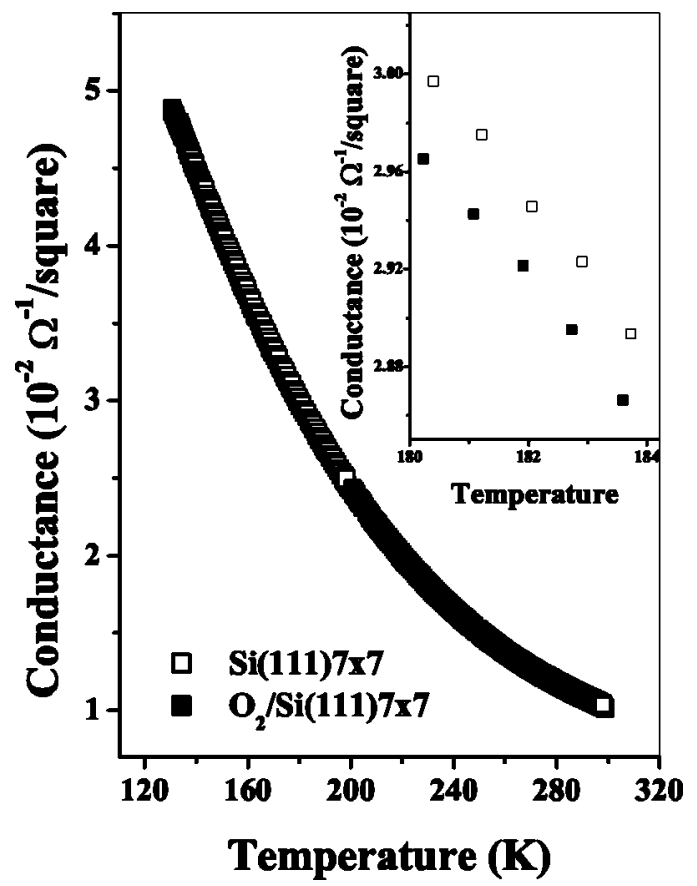

FIG. 10. Conductance of $\mathrm{Si}(111) 7 \times 7$ and oxidized $\mathrm{Si}(111)$ on SOI as a function of temperature. The Si layer is $200 \mathrm{~nm}$ thick. Inset: Enlarged scale showing the difference between the clean 7 $\times 7$ and the oxidized, flat band surface.

\section{SUMMARY AND CONCLUDING REMARKS}

The surface-state conductance of reconstructed Si surfaces was determined by comparing the electrical conductance on clean reconstructed $\mathrm{Si}$ and oxidized $\mathrm{Si}$ in situ. Because one cannot isolate the surface from the bulk, one cannot measure the surface-state conductance directly so a reference is needed. The oxidized surface served as a conve-

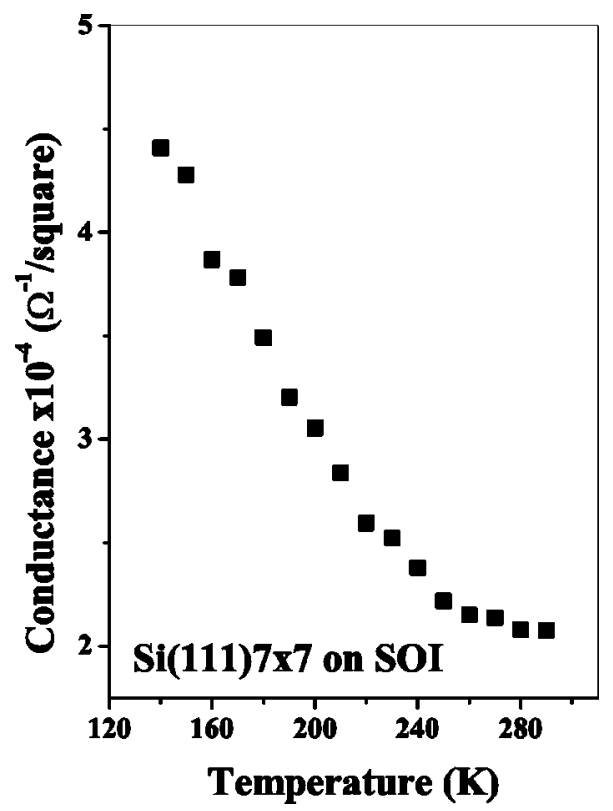

FIG. 11. Temperature dependence of surface-state conductance of $\mathrm{Si}(111) 7 \times 7$ on $200 \mathrm{~nm}$ SOI. nient reference because it has no surface states and no band bending. This indirect determination of the surface-state conductance is not elegant, but the results are consistent and the conclusions are very plausible. We considered three conduction channels, i.e., bulk, surface, and space-charge layer. Oxidation of the reconstructed surface always resulted in a few percent decrease of the total sheet conductance. This result cannot be explained in terms of band bending or adsorbate-induced surface scattering. Instead, the results are fully consistent with the presence of a surface-state conduction channel that is gradually destroyed by the oxidation process. The surface-state conductance of $\mathrm{Si}(100) 2 \times 1$ and $\operatorname{Si}(111) 7 \times 7$ exhibits metallic temperature dependence although the absolute magnitude of the conductance of $\mathrm{Si}(100)$ is comparable to (bulk) or even less (SOI) than the universal conductance quantum. The conductance of $\mathrm{Si}(100)$ appears to correlate with the degree of $c(4 \times 2)$ order on the surface. This work thus establishes a correlation between the atomic scale nature of a surface and a macroscopic materials parameter, such as the conductivity. Many other surface reconstructions can be considered as new materials that are intrinsically 2D and hence their transport properties will be of great fundamental and possibly practical interest.

It would be worthwhile to verify the conclusions and propositions of this paper by, e.g., using different adsorbates to tune the surface potential, such as, hydrogen. To enhance the surface sensitivity, one could also think of producing better insulating SOI materials. Perhaps a more fruitful approach would be to reduce the probe spacing to submicron distances $^{31}$ or even employ a four-point probe STM stage. ${ }^{32}$ Reduced probe spacing naturally enhances the surface sensitivity, but temperature-dependent measurements are difficult with these setups. Very small probe spacings and very low temperatures will probably yield quantitatively more reliable estimates of the surface-state conductance as the parallel conduction channel is more suppressed. It will remain a challenge to conduct such mesoscopic physics experiments in UHV environments.

\section{ACKNOWLEDGMENTS}

We thank F. Namavar for providing the (111)SOI wafers. We also acknowledge D. M. Zehner, J. J. Jellison, A. P. Baddorf, D. K. Thomas, and P. M. Fleming for their technical support. This work was primarily funded by the National Science Foundation under Contract No. DMR 9705246. Additional support was provided by the Laboratory Directed Research and Development Program of Oak Ridge National Laboratory, managed by UT-Battelle, LLC, for the U.S. Department of Energy under Contract No. DE-AC-0500OR22725.

\section{APPENDIX}

The physics of space-charge layers is, of course, not new. However, for the present purposes we need to evaluate the temperature dependence of the space-charge layer conductance. We begin with the definition of the surface conductance $\Delta \sigma$ of a bulk sample, ${ }^{20}$ 
TABLE I. Input parameters for the space-charge layer calculations at two different temperatures. The densities of the majority carriers were obtained from Hall effect measurements and the minority-carrier densities were calculated with the aid of Ref. 36.

\begin{tabular}{lcc}
\hline \hline Temperature $(\mathrm{K})$ & 300 & 120 \\
\hline$p_{b}\left(\mathrm{~cm}^{-3}\right)$ & $9.5 \times 10^{14}$ & $9.4 \times 10^{14}$ \\
$n_{b}\left(\mathrm{~cm}^{-3}\right)$ & $2.4 \times 10^{5}$ & $2.0 \times 10^{-26}$ \\
$u_{b}$ & 11.1 & 46.9 \\
$L_{D}(\mathrm{~cm})$ & $1.3 \times 10^{-5}$ & $8.4 \times 10^{-6}$ \\
$E_{g}(\mathrm{eV})$ & 1.12 & 1.16 \\
\hline \hline
\end{tabular}

$$
G(T)=\sigma_{b}(T) d+\Delta \sigma(T)
$$

where $G(T)$ is the square conductance of the sample in $\Omega^{-1} / \square$, and $\sigma_{b}(T)$ the conductivity of the bulk and $d$ the thickness of the sample. $\Delta \sigma(T)$ includes electronic conduction through all surface channels, such as surface states or interface states, space-charge layer, and overlayers (if any). Our main interest is to determine the conductance intrinsic to the surface states. To determine the surface-state conductance from a measurement of the total sheet conductance, we need to subtract the bulk conductance and space-charge-layer conductance $\Delta \sigma_{s c}(T)$. Following Greene, we have $\mathrm{e}^{20}$

$$
\Delta \sigma_{s c}=e\left(\mu_{n, s} \Delta N+\mu_{p, s} \Delta P\right),
$$

where $\Delta N$ and $\Delta P$ are the excess densities of electrons $(n)$ and holes $(p)$, respectively, near the surface; $\mu_{n, s}$ and $\mu_{p, s}$ are the surface mobilities, which are defined by this equation. They should not be interpreted as ordinary drift mobility.

\section{Surface excess carrier density}

Depending on the sign and magnitude of the band bending, we can distinguish between depletion, accumulation, and inversion layers. For $\mathrm{Si}(100) 2 \times 1$ and $\mathrm{Si}(111) 7 \times 7$, we are dealing with a depletion layer. Depletion layers are depleted of mobile carriers $(\Delta N, \Delta P<0)$ and therefore have a reduced conductance compared to the bulk.

The excess carrier density per unit surface area can be calculated from

$$
\begin{aligned}
& \Delta N=\int_{o}^{\infty}\left[n(z)-n_{b}\right] d z, \\
& \Delta P=\int_{o}^{\infty}\left[p(z)-p_{b}\right] d z,
\end{aligned}
$$

where $\Delta N$ and $\Delta P$ are the excess densities of electrons $(n)$ and holes $(p)$, respectively, near the surface. Subscripts $s$ and $b$ refer to the surface $(z=0)$ and bulk $(z=\infty)$, respectively. By solving Poisson's equation in the abrupt depletion approximation using Boltzmann statistics and parabolic bands, and introducing the new variables $v(z) \equiv\left[E_{i b}-E_{i}(z)\right] / k_{B} T$ and $u(z) \equiv\left[E_{F}-E_{i}(z)\right] / k_{B} T$, Kingston and Neustadter derived $^{33}$

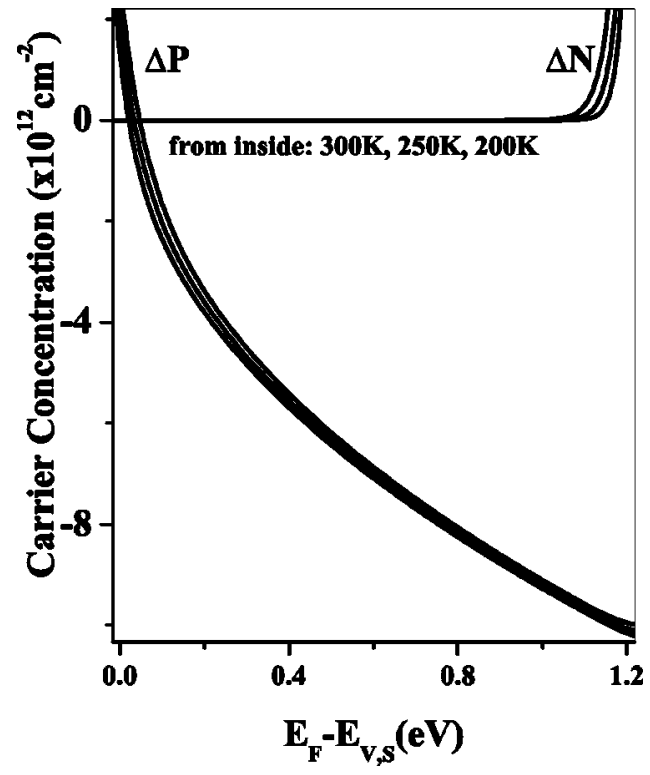

FIG. 12. Carrier concentration in Si space-charge layers as a function of band bending at 300,250 , and $200 \mathrm{~K}$ from the middle of the figure.

$$
\begin{gathered}
\Delta N=n_{b} L_{D} \int_{v_{s}}^{0}\left(e^{v}-1\right) / \mp F\left(u_{b}, v\right) d v \\
\Delta P=p_{b} L_{D} \int_{v_{s}}^{0}\left(e^{-v}-1\right) / \mp F\left(u_{b}, v\right) d v \\
F\left(u_{b}, v\right)=\sqrt{2} \sqrt{\left[\cosh \left(u_{b}+v\right) / \cosh u_{b}-v \tanh u_{b}-1\right]}, \\
L_{D}=\sqrt{\frac{\epsilon_{b} \epsilon_{0} k_{b} T}{e^{2}\left(n_{b}+p_{b}\right)}}
\end{gathered}
$$

where $E_{i}(z)$ is the intrinsic Fermi level, $v(z)$ the band bending $\left[v(0)=v_{s}\right], L_{D}$ the extrinsic Debije length, and $k_{B}$

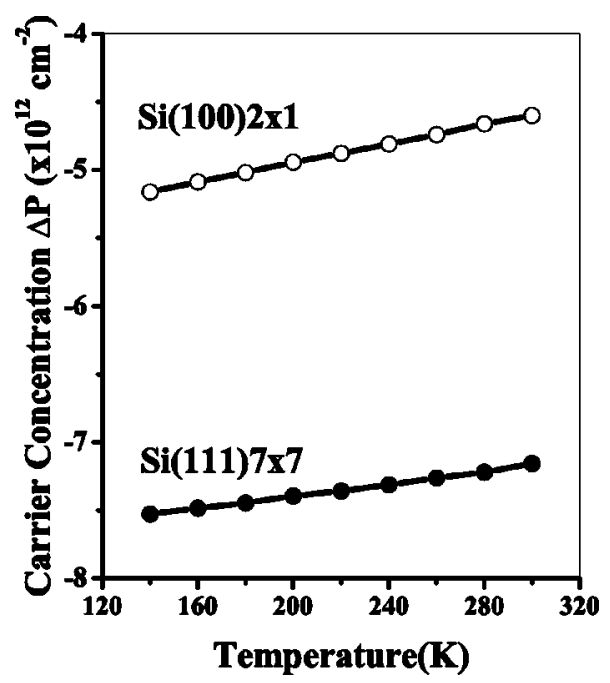

FIG. 13. Carrier concentration in the Si space-charge layer of $\mathrm{Si}(100) 2 \times 1$ and $\mathrm{Si}(111) 7 \times 7$ as a function of temperature. 


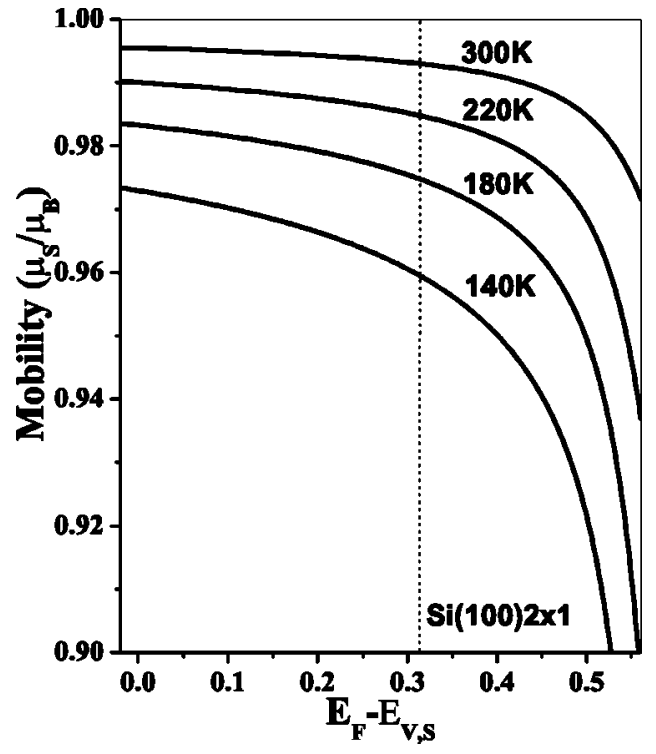

FIG. 14. Surface mobility in Si space-charge layers as a function of the band bending at different temperatures, assuming $r=0$.

Boltzmann's constant. The notation differs from the original notation in Ref. 33. Instead, we used the notation by Mönch. ${ }^{34}$ To perform the calculations for $p$-type $\operatorname{Si}(100) 2$ $\times 1$ and $\operatorname{Si}(111) 7 \times 7$, we used the parameters listed in Table I. By the way, the calculations for Fig. 6 were done for $n$-type Si. Calculations were done as a function of temperature by taking into account the temperature dependence of the parameters $L_{D}, v_{s}, u_{b}$, and $p_{b}$. The only parameter that is specific to the surface is the band bending $v_{s}$. It can be evaluated from the position of the Fermi level relative to the valence band maximum (VBM) at the surface, which is assumed to be independent of temperature due to "Fermi level

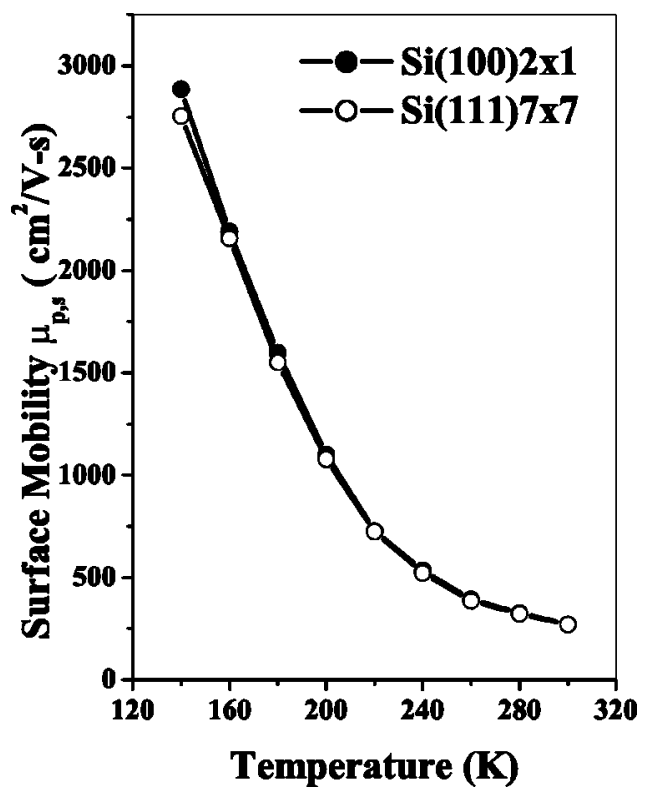

FIG. 15. Surface mobility in space-charge layer of $\mathrm{Si}(100) 2$ $\times 1$ and $\operatorname{Si}(111) 7 \times 7$ as a function of temperature, assuming $r$ $=0$.

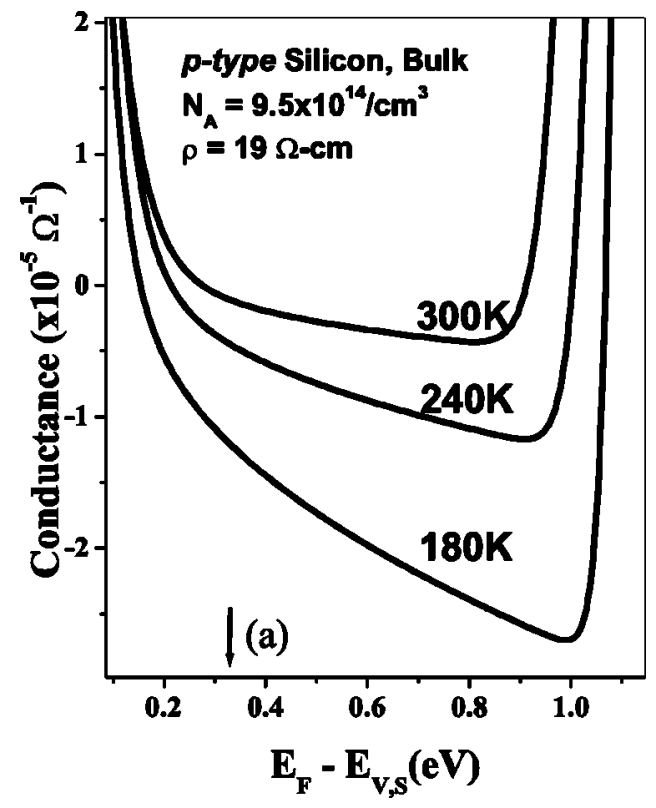

FIG. 16. Space-charge layer conductance as a function of band bending at three different temperatures. (a) indicates the Fermi level position at the $\operatorname{Si}(100) 2 \times 1$ surface.

pinning." For $\operatorname{Si}(100) 2 \times 1$ and $\operatorname{Si}(111) 7 \times 7$, we use $\left(E_{F}\right.$ $\left.-E_{V B M}\right)=0.31 \mathrm{eV}$ and $0.65 \mathrm{eV},{ }^{22}$ respectively. The total band bending $v_{s}$, however, does dependent on temperature because the Fermi level in the neutral bulk changes moves closer to the band edge as the temperature is lowered. The integrals (A5) and (A6) were evaluated using MATLAB and the results are shown in Figs. 12 and 13.

\section{Surface mobility}

A correct treatment of the mobility in space-charge layers is performed in two steps. First, the Boltzmann transport equation is solved taking into account the transverse electric

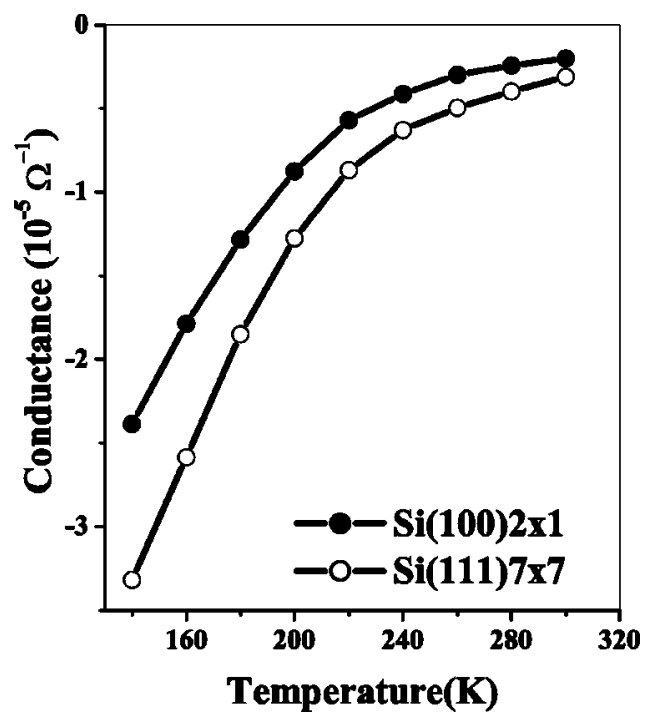

FIG. 17. Space-charge layer conductance of $\mathrm{Si}(100) 2 \times 1$ and $\operatorname{Si}(111) 7 \times 7$ as a function of temperature, assuming $r=0$. 
field associated with the space-charge layer. Next, surface scattering must be included. This can be done phenomenologically using the Fuchs scattering parameter $r$, which represents the fraction of specular scattering events. ${ }^{25}$ Greene has worked out the solution. ${ }^{20}$ Here, we use the approximate solution of the Boltzmann equation for depletion layers, which ignores minority carriers, but is accurate within $1 \%,{ }^{35}$

$$
\begin{gathered}
\mu_{s}=\mu_{b}\left\{1-\frac{M_{s} \lambda_{b}}{L_{D}}\right\}, \\
M_{s}=\frac{1}{\sqrt{2}}\left\{\frac{1-e^{-\left|v_{s}\right|}}{\sqrt{\left(e^{\left.-\left|v_{s}\right|+\left|v_{s}\right|-1\right)}\right.}}\right\},
\end{gathered}
$$

where $\mu_{b}$ is the bulk mobility of the majority carriers, $\lambda_{b}$ $\approx\left(\mu_{n, b} / e\right) \sqrt{\left(3 m^{*} k_{B} T\right)}$ the bulk mean free path. Surface scattering can be taken into account by introducing the Fuchs' parameter $r^{25}$

$$
\mu_{s}=\mu_{b}\left\{1-\frac{(1-r) M_{s} \lambda_{b}}{L_{D} \sqrt{2}}\right\} .
$$

Figure 14 shows the surface mobility as a function of the surface potential $v_{s}$ in the case of diffusive surface scattering, that is, $r=0$. In the above calculations, the temperaturedependent surface mobility for $\mathrm{Si}(100) 2 \times 1$ is obtained from the intersections of the vertical dotted line $\left(E_{F}-E_{V B M}\right.$ $=0.31 \mathrm{eV})$ (Ref. 9) and the different mobility isotherms.
The figure shows that the surface mobility in the depletion layer is reduced compared to the bulk value in the case of $\mathrm{Si}(100)$, the reduction is only on the order of a few percent. This reduction is even zero if we take $r=1\left(\mu_{s}=\mu_{b}\right)$. The Fuchs' parameter $r$ depends on the microscopic nature of the surface scattering mechanism, such as Coulomb scattering by ionized adsorbates, or scattering due to surface roughness, surface phonons, etc., and should also be temperature dependent. Our lack of knowledge regarding these scattering mechanisms has no severe consequences for the calculation of the space-charge layer mobility because $0 \leqslant r(T) \leqslant 1$, and hence, the mobility corrections to $\Delta \sigma(T)$ always remain within a few percent. Figure 15 shows the surface mobility of $\mathrm{Si}(100) 2 \times 1$ and $\mathrm{Si}(111) 7 \times 7$ as a function of temperature. Figures 16 and 17 show the final results of the spacecharge layer conductance as a function of band bending and temperature for $\mathrm{Si}(111) 7 \times 7$ and $\mathrm{Si}(100) 2 \times 1$ assuming $r$ $=0$. The space-charge layer conductance becomes increasingly negative at lower temperatures, which can be mostly attributed to the change in the carrier density. As the temperature decreases, the bulk conduction band edge moves closer to the Fermi level, i.e., band bending increases. Consequently, the space charge layer becomes increasingly depleted of mobile carriers. Once more, the underlying assumptions are that the Boltzmann statistics remains applicable in this temperature range and that the Fermi level is pinned at the surface.
${ }^{1}$ G. Bergmann, Phys. Rep. 107, 1 (1984).

${ }^{2}$ S. Hasegawa and S. Ino, Phys. Rev. Lett. 68, 1192 (1992).

${ }^{3}$ Y. Hasegawa, I.-W. Lyo, and P. Avouris, Surf. Sci. 357-358, 32 (1996).

${ }^{4}$ S. Heike, S. Watanabe, Y. Wada, and T. Hashizume, Phys. Rev. Lett. 81, 890 (1998).

${ }^{5}$ M. Jalochowski and E. Bauer, Phys. Rev. B 37, 8622 (1988).

${ }^{6}$ M. Jalochowski, M. Hoffmann, and E. Bauer, Phys. Rev. B 51, 7231 (1995).

${ }^{7}$ R. Schad, S. Heun, T. Heidenblut, and M. Henzler, Phys. Rev. B 45, 11430 (1992).

${ }^{8}$ M. Henzler, O. Pfennigtorf, K. Lang, T. Luer, F. Moresco, and T. Hildebrandt, Surf. Sci. 438, 178 (1999).

${ }^{9}$ K. Yoo and H.H. Weitering, Phys. Rev. Lett. 87, 026802 (2001).

${ }^{10}$ K.C. Lin, O.W. Holland, L.C. Feldman, and H.H. Weitering, Appl. Phys. Lett. 72, 2313 (1998).

${ }^{11}$ M. Noh, G.E. Jellison, Jr., F. Namavar, and H.H. Weitering, Appl. Phys. Lett. 76, 733 (2000).

${ }^{12}$ C.B. Duke, Chem. Rev. 96, 1237 (1996), and references cited therein.

${ }^{13}$ R.J. Hamers and U.K. Kohler, J. Vac. Sci. Technol. A 7, 2854 (1989).

${ }^{14}$ J. Ortega, F. Flores, and A.L. Yeyati, Phys. Rev. B 58, 4584 (1998).

${ }^{15}$ R. Losio, K.N. Altmann, and F.J. Himpsel, Phys. Rev. B 61, 10 845 (2000).
${ }^{16}$ F.J. Himpsel, G. Hollinger, and R.A. Pollak, Phys. Rev. B 28, 7014 (1983).

${ }^{17}$ J.P. Colinge, Silicon-on-Insulator Technology: Materials to VLSI, 2nd ed. (Kluwer Academic, Norwell, MA, 1997).

${ }^{18}$ D.I. Siapkas, N. Hatzopoulos, C.C. Katsidis, T. Zorba, C.L. Mitsas, and P.L.F. Hemment, J. Electrochem. Soc. 143, 3019 (1996).

${ }^{19}$ Y. Ono, M. Nagase, M. Tabe, and Y. Takahashi, Jpn. J. Appl. Phys., Part 1 34, 1728 (1995).

${ }^{20}$ R.F. Greene, CRC Crit. Rev. Solid State Sci. 4, 477 (1974).

${ }^{21}$ H. Ikegami, K. Ohmori, H. Ikeda, H. Iwano, S. Zaima, and Y. Yasuda, Jpn. J. Appl. Phys., Part 1 35, 1593 (1996).

${ }^{22}$ G. Hollinger and F.J. Himpsel, J. Vac. Sci. Technol. A 1, 640 (1983).

${ }^{23}$ H. Wormeester, E.G. Keim, and A. van Silfhout, Surf. Sci. 271, 340 (1992).

${ }^{24}$ C.L. Petersen, F. Grey, and M. Aono, Surf. Sci. 377-379, 676 (1997).

${ }^{25}$ E.H. Sondheimer, Adv. Phys. 1, 1 (1952).

${ }^{26}$ From ellipsometry measurements the refractive index of the buried oxide layer was 1.612 , which compares to 1.46 for pure stoichiometric silica. The substoichiometric oxide in SOI results in poor electrical insulation between top and bottom $\mathrm{Si}$.

${ }^{27}$ M. Kubota and Y. Murata, Phys. Rev. B 49, 4810 (1994). The definition of $T_{c}$ is somewhat arbitrary. It ranges from $\sim 200$ to $\sim 250 \mathrm{~K}$ depending on the defect density. See also, K. Inoue et al., ibid. 49, 14774 (1994).

${ }^{28}$ D.K. Ferry and S.M. Goodnick, Transport in Nanostructures 
(Cambridge University Press, Cambridge, UK, 1997).

${ }^{29}$ S.B. Arnason, S.P. Herschfield, and A.F. Hebard, Phys. Rev. Lett. 81, 3936 (1998).

${ }^{30}$ L. Klein, J.S. Dodge, C.H. Ahn, G.J. Snyder, T.H. Geballe, M.R. Beasley, and A. Kapitulnik, Phys. Rev. Lett. 77, 2774 (1996).

${ }^{31}$ C.L. Petersen, F. Grey, I. Shiraki, and S. Hasegawa, Appl. Phys. Lett. 77, 3782 (2000).

${ }^{32} \mathrm{~S}$. Hasegawa (unpublished).
${ }^{33}$ R.H. Kingston and S.F. Neustadter, J. Appl. Phys. 26, 718 (1955).

${ }^{34}$ W. Mönch, Semiconductor Surfaces and Interfaces, 2nd ed., Springer Series in Surface Sciences (Springer, Berlin, 1995).

${ }^{35}$ Y. Goldstein, N.B. Grover, A. Many, and R.F. Greene, J. Appl. Phys. 32, 2540 (1961).

${ }^{36}$ S.M. Sze, Physics of Semiconductor Devices, 2nd ed. (J. Wiley, New York, 1981). 